\title{
Assessment of pollutant elements content in ambient air dust of Khuzestan province
}

\author{
Z. Akbari ${ }^{1}$, O. Kakuee ${ }^{1 *}$, R. Shahbazi ${ }^{2}$, J. Darvishi Khatooni ${ }^{3}$, M. Mashal ${ }^{4}$ \\ ${ }^{1}$ Physics \& Accelerators Research School, Nuclear Science and Technology Research Institute, 14395-836 Tehran, Iran. \\ ${ }^{2}$ Director Management of Geohazards, Engineering and Environmental Geology, Tehran, Iran. \\ ${ }^{3}$ Geological Survey of Iran. \\ ${ }^{4}$ Geological Survey of Iran, Southwestern Area (Ahvaz Center), Ahvaz, Iran,
}

\begin{abstract}
This study aimed to investigate the distribution of natural and anthropogenic pollutants and the enrichment of elements in air dust of Khuzestan province following the dust events. Dust samples were collected from nine regions including, Abadan, Ahvaz, Hoveyzeh, Susangerd, Shush, Omidieh, Ramhormoz, and Mahshahr. The INAA technique measured the concentration of elements. Using the results of PMF modeling and investigation of obtained factors for samples, a suitable reference element with the most negligible influence from pollutant sources was selected and used for EF calculations. The results showed very large enrichments with EF> 20 for elements such as Zn, Se, Br in Susangerd, Ahvaz, and Abadan. The concentrations of Fe, Al, and Mg in some areas of the province were much higher than LC50. The enrichment factors and the correlations between the elements in the samples of various regions showed their dependence on local pollutant sources.
\end{abstract}

Keywords: Dust, Enrichment Factor, LC50, Pollutants, INAA

\section{INTRODUCTION}

Khuzestan province on the west coast of Iran is one of the most populated areas due to its fertile plain. Although much dust in the Khuzestan province derives from local sources, substantial amounts come from the transboundary such as Sahara [1]. Moreover, seasonal dust is one of the most critical issues in the Middle East, especially in recent years in Khuzestan province [2-9]. More than 30\% of Khuzestan province is covered with clay belts [10]. Due to clay particles' geometric and geochemical structure, they are strong absorbers of chemical elements such as metals, especially heavy metals. Therefore, the presence of clay particles in the dust causes the displacement of air pollutants. In
Khuzestan province, there are many air pollutant sources, including oil and metal industries and power plants. In addition, dried wetlands such as Hoor-Al-Azim and Shadegan and the waste accumulated are important sources of natural and artificial pollutants in this province. (Table 1) shows The lethal concentration 50 (LC50) values; LC50 is the concentration of a chemical that will kill 50 percent of the sample population under scrutiny of the metallic elements for the respiratory system. These values are for inhalation in the air for four consecutive hours [17]. In similar studies, the effects of a dust event in the transmission of pollution elements due to industrial pollutant

\footnotetext{
*Coressponding Author: okakuee@ aeoi.org.ir
} 
sources were investigated [18-21]. In one of these works that have been done in China, distribution of pollution from pollutant sources and the impact of industrial activities on the heavy metal content of dust fall in the desert steppe is investigated; results have shown the transportation of industrial pollutions have had a significant impact on the heavy metal content of dust fall in the desert steppe[18].

In this study, by calculating LC50 and the levels of EF (Enrichment Factor) for the chemical elements in the dust samples, those elements with critical levels of pollution, especially metallic and toxic elements, and the regions with the most highrisk condition in the dust events in Khuzestan province, will be recognized. Calculation of PMF (Positive Matrix Factorization) modeling and correlation coefficients among the chemical elements obtained for samples will indicate the probable pollutant sources that affected the dust samples in the study region. The distribution of chemical elements all over the sampling area of Khuzestan province will confirm the impact of pollutant sources of dust samples.

\section{RESEARCH THEORIES}

Dust is mainly originated from surface soil. Therefore, through soil erosion in the atmospheric storms, dust enters the atmosphere, and the elements in the dust are enriched by absorbing the atmospheric pollutions.

\section{Table 1}

LC50 of the elements for 4 hours continuous inhalation (air density $1.225 \mathrm{~kg} / \mathrm{cm}^{3}$ ) [11-16].

\begin{tabular}{cccccc}
\hline Element & $\begin{array}{c}\mathrm{LC50} \\
\left(\mathrm{mg} / \mathrm{m}^{3}\right)\end{array}$ & $\begin{array}{c}L C 50 \\
(\mathrm{mg} / \mathrm{g})\end{array}$ & Element & $\begin{array}{c}L C 50 \\
\left(\mathrm{mg} / \mathrm{m}^{3}\right)\end{array}$ & $\begin{array}{c}L C 50 \\
(\mathrm{mg} / \mathrm{g})\end{array}$ \\
\hline $\mathrm{Fe}$ & 1100 & 0.90 & $\mathrm{Cr}$ & 5410 & 4.42 \\
$\mathrm{Mn}$ & 4450 & 3.63 & $\mathrm{Ti}$ & 6820 & 5.57 \\
$\mathrm{Co}$ & 50 & 0.041 & $\mathrm{Al}$ & 2300 & 1.88 \\
$\mathrm{Mg}$ & 2100 & 1.71 & $\mathrm{Zn}$ & 5700 & 4.65 \\
\hline
\end{tabular}

The quantity that is commonly used to study the level of enrichment of elements in geological and environmental samples is known as the enrichment factor [22]. This factor indicates the amount of change in the concentration of elements. The changes are mainly due to the effect of human resources.

Due to the innumerable distribution of air pollutant sources in Khuzestan province, the elements in dust samples are strongly affected by these sources. In most enrichment studies in surface soil samples, the reference material is usually soil, and the reference element is selected among the five elements $\mathrm{Al}, \mathrm{Mn}, \mathrm{Ti}, \mathrm{Fe}$, and $\mathrm{Sc}$ [23-25]; the reason for this selection is the low mobility of these elements, and their most negligible influences from polluting sources $[26,27]$. In the case of dust samples, soil can be used as the reference material. However, in the dust samples collected from different parts of the province, some of these elements, depending on the geographical location, are affected by the atmospheric pollutants. The concentration of the elements varies from their natural concentration in the soil. Therefore, not all of them are candidates for the reference element anymore. In this situation, the best solution is to perform a PMF modeling on the elemental concentrations measured in the samples to find possible influencing sources. Then, according to the elemental composition of the pollutions from pollutant sources in the study region and the resulted factors for each sample from PMF 
modeling, among the five elements mentioned, the most suitable with the least influence from the pollutant sources was used as the reference element [28-32].

The study of dust samples from different regions of southwestern Iran is of specific importance in investigating how industrial and natural airborne pollutants are distributed and the level of enrichment of elements in the dust.

The enrichment factor is defined by the following formula [33].

EnrichmentFactor $=\frac{\mathrm{C}_{\mathrm{e}_{\text {sample }} / \mathrm{C}_{\text {re_sample }}}}{\mathrm{C}_{\mathrm{e} \_ \text {ref }} / \mathrm{C}_{\text {re_ref }}}$

Ce_sample is the concentration of the element in the sample, Cre_sample is the concentration of the reference element in the sample, Ce_ref is the concentration of the element in the reference material, and Cre_ref is the reference element in the reference material. As mentioned in the introduction, the changes in the elemental concentrations of dust samples throughout the study area and how they change with location affect the reference selection. The reference material can be considered crustal soil that other natural and anthropogenic sources can disturb the natural soil concentrations in the samples. For example, $\mathrm{Br}$ and $\mathrm{Na}$, which mainly are raised from the seawater or the bed of wetlands and sediments in the study region, are a kind of disruptor for the concentration of these elements in the soil [34].

In the case of soil reference material, one of the major elements in the soil, such as $\mathrm{Sc}, \mathrm{Fe}, \mathrm{Al}, \mathrm{Ti}$, and $\mathrm{Mn}$, is selected as the reference element. These elements usually have less mobility than the others do. To choose the most appropriate one among these five elements for each sample, the best method is to implement a modeling technique such as PMF or PCA on the elemental concentrations of the samples. In this way, by assessing the obtained factors, it is possible to find out the sources affecting the samples; also, polluting sources have affected which of the mentioned elements and enriched them, and which of them are less affected [35]. Indeed, those affected minimally are the most appropriate option to choose as a reference element [36].

\section{EXPERIMENTAL}

A. Analysis of samples using (Instrumental Neutron Activation Analysis) INAA nuclear technique

Dust samples were collected in nine regions of Khuzestan province. Samples were collected three times in Abadan from August 2018 to January 2019, and the collection time was one month. The next sampling was in February 2020, in seven other regions, including Dezful, Shush, Susangerd, Hoveyzeh, Mahshahr, Omidieh, and Ramhormoz, and then in April 2020 in Ahvaz. The sampling was performed for one week in these cities. (Fig 1) shows the sampling points. Samples were collected in a glass box with dimensions of $70 \times 70 \times 20 \mathrm{~cm} 3$ on the roof of high buildings. There were no other high buildings around the sampling locations. The dust samples' grains, were mainly in clay size, and the maximum particle diameter was $4 \mu \mathrm{m}$.

The instrumental neutron activation analysis (INAA) technique was used for the elemental analysis of the samples. Dust samples were dried in 
the oven for around 12 hours and then weighted.

The maximum weight of the samples used was 250 $\mathrm{mg}$. The weighted dust samples were poured into the plastic covers, labeled, and put in the polyethylene capsules for irradiation. The standards used in this technique included IAEASOIL-7, IAEA-SOIL-5, and Coal-1632a. Five samples with the standard smaples were put in one capsule for long irradiation. For short irradiation one dust sample and one standard sample were put in each capsule. The samples were irradiated in the Tehran research reactor at the power of 4MW(Megawatts) in two categories; irradiation for elements with short half-lives and elements with long half-lives. Short irradiation time was 5 second; and long irradiation was 45 minutes.

The first group was counted immediately after irradiation and the second after a determined decay time. Short half-life radionuclides were counted using an HPGe detector with an efficiency of $15 \%$ and resolution of $2 \mathrm{keV}$ at $1332 \mathrm{keV}$ energy related to Co-60; mid and long half-life radionuclide were counted using an HPGe detector with an efficiency of $30 \%$ and resolution of $2 \mathrm{keV}$ at $1332 \mathrm{keV}$ energy related to Co-60. Eu-152 standard point source was used for energy calibration.

For analysis of gamma spectrum, SPAN software was used. The analysis technique in this software is based on the comparative method using the gamma spectrums of irradiated standard samples; therefore, there is no need to calculate the absolute efficiency of energy peaks. (Table 2), shows the measurable elements in the spectra and their uncertainties with the same units. In (Table 3) some specifications of obtained radionuclides are represented. In (Fig 2), three spectrums of the Abadan-Dec sample have been shown for short half-life, mid-halflife, and long-halflife radionuclides. The horizontal axis in the spectrum of the short half-life radionuclides represents the channel number, and the vertical axis represents the number of counts. For two other spectra, the horizontal axis represents the gamma energy. The main interference of gamma energies is about $843 \mathrm{keV}$ of $\mathrm{Mg}-27$ and $846 \mathrm{keV}$ of $\mathrm{Mn}-56$. Therefore, for these two radionuclides, their other gamma energies were used; for Mn-56 1810keV and for Mg-27 1014keV were used. The other main interference was about $277 \mathrm{keV}$ Nep-239; this radionuclide decays to U-238 and is used for uranium analysis. $\mathrm{Hg}-203$ has an energy of $279 \mathrm{keV}$. In dust samples amount of HG-203 is not considerable; however, for SOIL-7 standard, this element has noticeable value. Therefore, for analysis of Np-239 in dust samples, SOIL-5 standard was used. For other radionuclides with interfered gamma energy, it was also tried to use their other gamma energies with no interference.

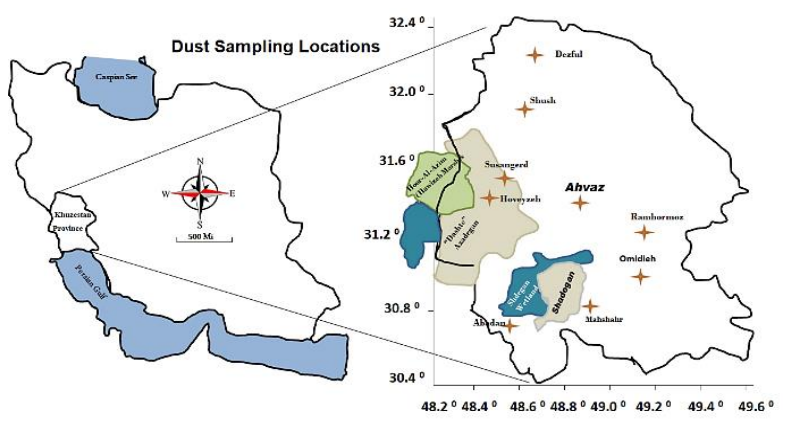

Fig.1 the sampling regions in Khuzestan province in southwestern Iran 
Table 2-a

Elemental concentration obtained from INAA analysis of dust samples

\begin{tabular}{|c|c|c|c|c|c|c|c|}
\hline Elements & unit & $\begin{array}{c}\text { Abadan } \\
(\text { Aug-2018) }\end{array}$ & $\begin{array}{c}\text { Abadan (Sep- } \\
\text { 2018) }\end{array}$ & $\begin{array}{c}\text { Abadan (Dec- } \\
\text { 2019) }\end{array}$ & $\begin{array}{c}\text { Ahvaz (Apr- } \\
\text { 2020) }\end{array}$ & $\begin{array}{c}\text { Dezful (Feb- } \\
\text { 2020) }\end{array}$ & $\begin{array}{c}\text { Hoveyzeh } \\
\text { (Feb-2020) }\end{array}$ \\
\hline Al & $\mathrm{g} / \mathrm{kg}$ & $26.96 \pm 4.53$ & $21.36 \pm 4.19$ & $38.34 \pm 6.21$ & $30.90 \pm 4.79$ & $39.13 \pm 6.22$ & $37.49 \pm 5.96$ \\
\hline As & $\mathrm{mg} / \mathrm{kg}$ & $7.80 \pm 3.30$ & BDL & BDL & $6.66 \pm 1.20$ & $5.40 \pm 1.00$ & $4.90 \pm 0.90$ \\
\hline $\mathbf{B a}$ & $\mathrm{mg} / \mathrm{kg}$ & $239.60 \pm 51.30$ & $361.60 \pm 74.50$ & $445.90 \pm 100.80$ & $425.400 \pm 71.50$ & $208.40 \pm 37.70$ & $223.70 \pm 42.50$ \\
\hline $\mathbf{B r}$ & $\mathrm{mg} / \mathrm{kg}$ & $24.70 \pm 4.00$ & $22.70 \pm 4.80$ & $8.70 \pm 2.80$ & $2.50 \pm 0.80$ & $3.50 \pm 0.80$ & $4.00 \pm 0.90$ \\
\hline $\mathrm{Ca}$ & $\mathrm{g} / \mathrm{kg}$ & $144.70 \pm 24.89$ & $186.63 \pm 31.91$ & $173.47 \pm 31.22$ & $176.27 \pm 28.73$ & $151.73 \pm 24.88$ & $177.28 \pm 29.25$ \\
\hline $\mathrm{Ce}$ & $\mathrm{mg} / \mathrm{kg}$ & $28.40 \pm 5.20$ & $67.80 \pm 13.60$ & $83.20 \pm 18.50$ & $34.80 \pm 5.70$ & $32.40 \pm 5.50$ & $36.30 \pm 6.00$ \\
\hline Co & $\mathrm{mg} / \mathrm{kg}$ & $12.10 \pm 2.10$ & $13.50 \pm 2.30$ & $13.40 \pm 2.40$ & $11.20 \pm 1.80$ & $10.20 \pm 1.60$ & $10.00 \pm 1.60$ \\
\hline $\mathrm{Cr}$ & $\mathrm{mg} / \mathrm{kg}$ & $88.80 \pm 17.70$ & $134.90 \pm 22.00$ & $134.20 \pm 23.50$ & $202.40 \pm 31.80$ & $229.30 \pm 35.90$ & $306.30 \pm 47.80$ \\
\hline Dy & $\mathrm{mg} / \mathrm{kg}$ & $2.40 \pm 0.20$ & BDL & $2.20 \pm 0.20$ & $2.50 \pm 0.60$ & $2.20 \pm 0.50$ & $2.40 \pm 0.50$ \\
\hline Eu & $\mathrm{mg} / \mathrm{kg}$ & (C) BDL & BDL & $0.60 \pm 0.10$ & $0.60 \pm 0.10$ & $0.60 \pm 0.20$ & $0.60 \pm 0.10$ \\
\hline $\mathbf{F e}$ & $\mathrm{g} / \mathrm{kg}$ & $23.48 \pm 3.78$ & $26.28 \pm 4.18$ & $42.26 \pm 6.80$ & $45.97 \pm 7.01$ & $46.15 \pm 7.06$ & $21.81 \pm 3.38$ \\
\hline Hf & $\mathrm{mg} / \mathrm{kg}$ & $2.80 \pm 0.60$ & $3.20 \pm 0.60$ & $4.10 \pm 0.80$ & $2.60 \pm 0.40$ & $3.20 \pm 0.50$ & $3.00 \pm 0.50$ \\
\hline $\mathbf{K}$ & $\mathrm{g} / \mathrm{kg}$ & BDL & BDL & BDL & $7.02 \pm 1.55$ & $6.81 \pm 1.58$ & $7.06 \pm 1.10$ \\
\hline La & $\mathrm{mg} / \mathrm{kg}$ & $19.30 \pm 3.20$ & $19.10 \pm 3.20$ & $15.20 \pm 2.80$ & $13.40 \pm 2.10$ & $14.60 \pm 2.30$ & $14.30 \pm 2.30$ \\
\hline $\mathbf{L u}$ & $\mathrm{mg} / \mathrm{kg}$ & $0.21 \pm 0.04$ & $0.23 \pm 0.04$ & $0.28 \pm 0.10$ & $0.15 \pm 0.02$ & $0.26 \pm 0.07$ & $0.10 \pm 0.02$ \\
\hline Mg & $\mathrm{g} / \mathrm{kg}$ & $19.68 \pm 3.35$ & $25.17 \pm 4.25$ & $17.43 \pm 3.10$ & $14.06 \pm 2.60$ & $18.02 \pm 3.26$ & $14.14 \pm 2.38$ \\
\hline Mn & $\mathrm{mg} / \mathrm{kg}$ & $507.70 \pm 85.50$ & $645.20 \pm 109.70$ & $564.30 \pm 93.70$ & $465.40 \pm 71.70$ & $343.50 \pm 53.60$ & $469.90 \pm 72.80$ \\
\hline $\mathbf{N a}$ & $\mathrm{g} / \mathrm{kg}$ & $35.52 \pm 5.56$ & $28.37 \pm 5.62$ & $5.83 \pm 0.95$ & $5.59 \pm 0.87$ & $3.69 \pm 0.57$ & $4.79 \pm 0.75$ \\
\hline Nd & $\mathrm{mg} / \mathrm{kg}$ & $12.50 \pm 3.70$ & $12.30 \pm 1.30$ & $298.20 \pm 50.10$ & $12.80 \pm 2.70$ & $15.50 \pm 3.10$ & $14.90 \pm 3.10$ \\
\hline $\mathbf{R b}$ & $\mathrm{mg} / \mathrm{kg}$ & $14.40 \pm 12.00$ & $41.10 \pm 10.40$ & $14.40 \pm 12.00$ & $23.30 \pm 5.40$ & $23.90 \pm 5.60$ & $27.00 \pm 6.10$ \\
\hline Sc & $\mathrm{mg} / \mathrm{kg}$ & $8.00 \pm 1.30$ & $8.50 \pm 1.30$ & $8.70 \pm 1.40$ & $6.40 \pm 1.00$ & $6.10 \pm 0.90$ & $7.40 \pm 1.10$ \\
\hline $\mathrm{Se}$ & $\mathrm{mg} / \mathrm{kg}$ & BDL & BDL & BDL & $4.10 \pm 1.50$ & $2.70 \pm 1.05$ & $0.38 \pm 0.10$ \\
\hline Sm & $\mathrm{mg} / \mathrm{kg}$ & $3.00 \pm 0.50$ & BDL & $3.30 \pm 0.60$ & $2.10 \pm 0.30$ & $2.50 \pm 0.40$ & $2.40 \pm 0.40$ \\
\hline $\mathbf{T a}$ & $\mathrm{mg} / \mathrm{kg}$ & $0.80 \pm 0.10$ & $1.30 \pm 0.40$ & $0.90 \pm 0.20$ & $0.30 \pm 0.10$ & $0.40 \pm 0.10$ & $0.30 \pm 0.10$ \\
\hline $\mathbf{T i}$ & $\mathrm{g} / \mathrm{kg}$ & $1.33 \pm 0.37$ & $2.61 \pm 0.61$ & $2.30 \pm 0.56$ & $3.55 \pm 0.65$ & $3.41 \pm 0.64$ & $2.12 \pm 0.46$ \\
\hline $\mathbf{v}$ & $\mathrm{mg} / \mathrm{kg}$ & $74.30 \pm 14.00$ & $97.20 \pm 17.90$ & $107.30 \pm 20.40$ & $120.40 \pm 19.90$ & $94.30 \pm 15.80$ & $73.90 \pm 13.40$ \\
\hline $\mathbf{Y b}$ & $\mathrm{mg} / \mathrm{kg}$ & $1.20 \pm 0.30$ & $1.40 \pm 0.40$ & $1.70 \pm 0.40$ & $1.00 \pm 0.20$ & $1.31 \pm 0.20$ & $1.20 \pm 0.20$ \\
\hline Zn & $\mathrm{mg} / \mathrm{kg}$ & $188.20 \pm 36.30$ & $404.40 \pm 69.60$ & $196.10 \pm 40.60$ & $227.30 \pm 37.70$ & $333.50 \pm 54.00$ & $134.60 \pm 23.30$ \\
\hline $\mathbf{U}$ & $\mathrm{mg} / \mathrm{kg}$ & $\mathrm{BDL}$ & $\mathrm{BDL}$ & $\mathrm{BDL}$ & $1.50 \pm 0.40$ & $1.60 \pm 0.40$ & $1.30 \pm 0.30$ \\
\hline Th & $\mathrm{mg} / \mathrm{kg}$ & BDL & BDL & BDL & $4.40 \pm 0.90$ & $3.20 \pm 0.60$ & $3.40 \pm 0.70$ \\
\hline
\end{tabular}


Table 2-b

\begin{tabular}{|c|c|c|c|c|c|c|}
\hline Elements & unit & $\begin{array}{c}\text { Mahshahr (Feb- } \\
\text { 2020) }\end{array}$ & $\begin{array}{l}\text { Omidieh (Feb- } \\
\text { 2020) }\end{array}$ & $\begin{array}{c}\text { Ramhormoz (Feb- } \\
\text { 2020) }\end{array}$ & $\begin{array}{c}\text { Shush (Feb- } \\
\text { 2020) }\end{array}$ & $\begin{array}{l}\text { Susangerd (Feb- } \\
\text { 2020) }\end{array}$ \\
\hline Al & $\mathrm{g} / \mathrm{kg}$ & $25.98 \pm 4.16$ & $33.66 \pm 5.15$ & $35.28 \pm 5.50$ & $42.64 \pm 6.65$ & $40.83 \pm 6.33$ \\
\hline As & $\mathrm{mg} / \mathrm{k}$ & $4.90 \pm 0.90$ & $6.40 \pm 1.20$ & $4.40 \pm 0.90$ & $5.70 \pm 1.10$ & $6.80 \pm 1.30$ \\
\hline $\mathbf{B a}$ & $\underset{\mathrm{mg} / \mathrm{k}}{\mathrm{g}}$ & $229.40 \pm 42.20$ & $212.30 \pm 38.40$ & $214.50 \pm 39.00$ & $181.20 \pm 35.50$ & $224.30 \pm 50.00$ \\
\hline Br & $\underset{\mathrm{mg} / \mathrm{k}}{\mathrm{g}}$ & $7.60 \pm 1.70$ & $3.20 \pm 0.80$ & $5.30 \pm 1.10$ & $3.90 \pm 0.90$ & $1.70 \pm 1.40$ \\
\hline & $\mathrm{g}$ & & & & & \\
\hline $\mathbf{C a}$ & $\mathrm{g} / \mathrm{kg}$ & $196.39 \pm 32.40$ & $205.46 \pm 33.90$ & $189.98 \pm 31.54$ & $138.54 \pm 23.14$ & $126.06 \pm 21.05$ \\
\hline $\mathrm{Ce}$ & $\mathrm{mg} / \mathrm{k}$ & $30.70 \pm 5.10$ & $25.80 \pm 4.30$ & $27.20 \pm 4.60$ & $36.80 \pm 6.10$ & $36.30 \pm 6.10$ \\
\hline Co & $\underset{\mathrm{mg} / \mathrm{k}}{\mathrm{g}}$ & $10.20 \pm 1.70$ & $7.30 \pm 1.20$ & $7.50 \pm 1.20$ & $10.00 \pm 1.60$ & $11.90 \pm 1.90$ \\
\hline $\mathrm{Cr}$ & $\underset{\mathrm{g} / \mathrm{kg}}{\mathrm{g}}$ & $222.60 \pm 35.00$ & $189.90 \pm 29.90$ & $133.90 \pm 21.40$ & $229.10 \pm 36.20$ & $235.20 \pm 37.20$ \\
\hline Dy & $\mathrm{mg} / \mathrm{k}$ & $0.90 \pm 0.30$ & $2.50 \pm 0.60$ & $2.30 \pm 0.50$ & $3.00 \pm 0.60$ & $3.70 \pm 0.80$ \\
\hline $\mathbf{E u}$ & $\underset{\mathrm{mg} / \mathrm{k}}{\mathrm{g}}$ & $0.60 \pm 0.10$ & $0.70 \pm 0.20$ & $0.60 \pm 0.20$ & $0.70 \pm 0.20$ & $0.90 \pm 0.20$ \\
\hline $\mathbf{F e}$ & $\underset{\mathrm{g} / \mathrm{kg}}{\mathrm{g}}$ & $34.00 \pm 5.24$ & $17.51 \pm 2.71$ & $17.94 \pm 2.80$ & $22.69 \pm 3.54$ & $36.06 \pm 5.55$ \\
\hline Hf & $\mathrm{mg} / \mathrm{k}$ & $2.50 \pm 0.40$ & $2.10 \pm 0.40$ & $2.20 \pm 0.40$ & $2.90 \pm 0.50$ & $3.20 \pm 0.50$ \\
\hline $\mathbf{K}$ & $\mathrm{g}$ & $4.36 \pm 1.08$ & $8.53 \pm 2.20$ & $8.95 \pm 1.95$ & $9.78 \pm 2.06$ & $8.41 \pm 1.80$ \\
\hline La & $\mathrm{mg} / \mathrm{k}$ & $12.50 \pm 2.00$ & $11.10 \pm 1.80$ & $11.20 \pm 1.80$ & $15.00 \pm 2.40$ & $15.00 \pm 2.40$ \\
\hline Lu & $\underset{\mathrm{mg} / \mathrm{k}}{\mathrm{g}}$ & $0.17 \pm 0.04$ & $0.17 \pm 0.05$ & $0.19 \pm 0.04$ & $0.27 \pm 0.05$ & $0.24 \pm 0.06$ \\
\hline Mg & $\underset{\mathrm{g} / \mathrm{kg}}{\mathrm{g}}$ & $13.97 \pm 2.74$ & $14.84 \pm 2.91$ & $13.74 \pm 2.29$ & $15.18 \pm 2.56$ & $16.69 \pm 2.79$ \\
\hline Mn & $\mathrm{mg} / \mathrm{k}$ & $291.50 \pm 46.90$ & $469.90 \pm 72.40$ & $342.60 \pm 54.10$ & $467.90 \pm 72.10$ & $523.60 \pm 81.20$ \\
\hline $\mathbf{N a}$ & $\underset{\mathrm{g} / \mathrm{kg}}{\mathrm{g}}$ & $8.14 \pm 1.27$ & $5.61 \pm 0.89$ & $6.54 \pm 1.02$ & $5.18 \pm 0.81$ & $6.05 \pm 0.94$ \\
\hline Nd & $\mathrm{mg} / \mathrm{k}$ & $12.30 \pm 2.90$ & $10.50 \pm 2.40$ & $10.60 \pm 2.30$ & $14.40 \pm 3.00$ & $17.20 \pm 3.60$ \\
\hline $\mathbf{R b}$ & $\underset{\mathrm{mg} / \mathrm{k}}{\mathrm{g}}$ & $29.30 \pm 7.00$ & $22.50 \pm 5.00$ & $19.80 \pm 4.60$ & $33.60 \pm 7.50$ & $32.80 \pm 7.30$ \\
\hline & $\mathrm{g}$ & & & & & \\
\hline Sc & $\mathrm{mg} / \mathrm{k}$ & $7.60 \pm 1.20$ & $6.70 \pm 1.00$ & $6.10 \pm 0.90$ & $6.50 \pm 1.00$ & $6.70 \pm 1.00$ \\
\hline Se & $\underset{\mathrm{mg} / \mathrm{k}}{\mathrm{g}}$ & BDL & BDL & $0.28 \pm 0.10$ & $0.27 \pm 0.10$ & $0.30 \pm 0.11$ \\
\hline & $\mathrm{g}$ & & & & & \\
\hline Sm & $\mathrm{mg} / \mathrm{k}$ & $2.20 \pm 0.40$ & $1.90 \pm 0.30$ & $1.80 \pm 0.30$ & $2.60 \pm 0.40$ & $2.70 \pm 0.40$ \\
\hline Ta & $\underset{\mathrm{mg} / \mathrm{k}}{\mathrm{g}}$ & $0.35 \pm 0.11$ & $0.25 \pm 0.15$ & $0.34 \pm 0.10$ & $0.42 \pm 0.11$ & $0.42 \pm 0.12$ \\
\hline & g & & & & & \\
\hline $\mathbf{T i}$ & $\mathrm{g} / \mathrm{kg}$ & $1.84 \pm 0.44$ & $1.35 \pm 0.33$ & $2.11 \pm 0.45$ & $2.81 \pm 0.56$ & $3.07 \pm 0.61$ \\
\hline $\mathbf{V}$ & $\mathrm{mg} / \mathrm{k}$ & $59.80 \pm 11.20$ & $126.90 \pm 22.20$ & $66.10 \pm 11.70$ & $99.90 \pm 17.10$ & $86.20 \pm 14.90$ \\
\hline $\mathbf{Y b}$ & $\underset{\mathrm{mg} / \mathrm{k}}{\mathrm{g}}$ & $0.90 \pm 0.20$ & $0.90 \pm 0.20$ & $0.95 \pm 0.23$ & $1.35 \pm 0.30$ & $1.31 \pm 0.30$ \\
\hline & $\mathrm{g}$ & & & & & \\
\hline Zn & $\mathrm{g} / \mathrm{kg}$ & $0.62 \pm 0.10$ & $0.17 \pm 0.03$ & $0.47 \pm 0.08$ & $0.13 \pm 0.02$ & $1.06 \pm 0.17$ \\
\hline $\mathbf{U}$ & $\mathrm{mg} / \mathrm{k}$ & $1.60 \pm 0.40$ & $1.70 \pm 0.42$ & $1.73 \pm 0.41$ & $1.40 \pm 0.38$ & $1.30 \pm 0.36$ \\
\hline Th & $\begin{array}{c}\mathrm{g} \\
\mathrm{mg} / \mathrm{k} \\
\mathrm{g}\end{array}$ & $2.70 \pm 0.60$ & $3.31 \pm 0.60$ & $3.30 \pm 0.62$ & $4.60 \pm 0.90$ & $4.60 \pm 0.95$ \\
\hline
\end{tabular}

(C) Below Detection Limit 


\section{Table 3}

Some Specifications of represented radionuclides in table 2

\begin{tabular}{|c|c|c|c|c|c|c|c|c|c|c|c|}
\hline \multicolumn{4}{|c|}{ Long-halflife } & \multicolumn{4}{|c|}{ Mid-Halflife } & \multicolumn{4}{|c|}{ Short-Halflife } \\
\hline 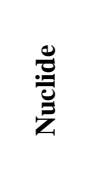 & 莺 & 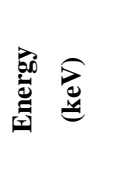 & 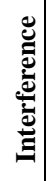 & 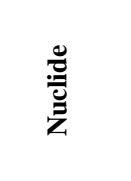 & 竎 & 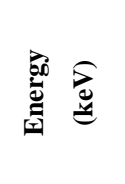 & 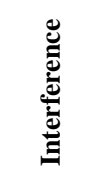 & 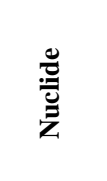 & 莺 & 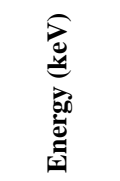 & 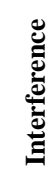 \\
\hline Sc-46 & $83.81 \mathrm{~d}$ & 889.28 & & $\mathrm{Na}-24$ & $14.96 \mathrm{~h}$ & 1368.60 & & Mg-27 & $9.46 \mathrm{~m}$ & 1014.43 & \\
\hline Sc-47 & $3.35 \mathrm{~d}$ & 159.38 & & Mn-56 & $2.58 \mathrm{~h}$ & 1810.72 & & Al-28 & $2.24 \mathrm{~m}$ & 1778.99 & \\
\hline Cr-51 & $27.7 \mathrm{~d}$ & 320.08 & & K-42 & $12.36 \mathrm{~h}$ & 1524.58 & & V-52 & $3.75 \mathrm{~m}$ & 1434.08 & \\
\hline Fe-59 & $44.5 \mathrm{~d}$ & 1099.25 & & Dy-165 & $2.33 \mathrm{~h}$ & 94.70 & & Ti-51 & $5.76 \mathrm{~m}$ & 320.08 & \\
\hline Co-60 & $5.27 \mathrm{yr}$ & 1173.24 & & Hf-180m & $5.52 \mathrm{~h}$ & 332.3 & & Ca-49 & $8.72 \mathrm{~m}$ & 3084.54 & \\
\hline Rb-86 & $18.66 \mathrm{~d}$ & 1076.60 & & Sm-153 & $46.27 \mathrm{~h}$ & 103.18 & & & & & \\
\hline Ce-141 & $32.5 \mathrm{~d}$ & 145.44 & & Br-82 & $35.3 \mathrm{~h}$ & 776.52 & & & & & \\
\hline Hf-181 & $42.39 \mathrm{~d}$ & 482.18 & & Np-239 & $2.36 \mathrm{~d}$ & 277.60 & $\mathrm{Hg}-203$ & & & & \\
\hline Se-75 & $119.77 \mathrm{~d}$ & 264.66 & & As-76 & $26.32 \mathrm{~h}$ & 559.10 & & & & & \\
\hline Eu-154 & $8.59 \mathrm{Yr}$ & 1274.51 & & Ba-139 & $84.63 \mathrm{~m}$ & 165.85 & & & & & \\
\hline Ta-182 & $114.5 \mathrm{~d}$ & 1221.41 & & $\begin{array}{c}\text { Ba- } \\
\text { 133m }\end{array}$ & $38.9 \mathrm{~h}$ & 275.93 & & & & & \\
\hline Nd-147 & $10.98 \mathrm{~d}$ & 531.01 & & $\begin{array}{c}\text { Eu- } \\
\text { 152m }\end{array}$ & $9.32 \mathrm{~h}$ & 121.78 & Mn-56 & & & & \\
\hline $\mathrm{Ca}-47$ & $4.54 \mathrm{~d}$ & 1297.09 & & La-140 & $40.27 \mathrm{~h}$ & 1596.21 & & & & & \\
\hline Mn-54 & $312.12 \mathrm{~d}$ & 834.84 & & & & & & & & & \\
\hline Zn-65 & $243.9 \mathrm{~d}$ & 1115.55 & & & & & & & & & \\
\hline Yb-169 & $32.02 \mathrm{~d}$ & 177.21 & & & & & & & & & \\
\hline $\begin{array}{c}\text { Lu- } \\
\text { 177m }\end{array}$ & $160.9 \mathrm{~d}$ & 208.86 & & & & & & & & & \\
\hline Pa-233 & $27 d$ & 312.01 & & & & & & & & & \\
\hline
\end{tabular}



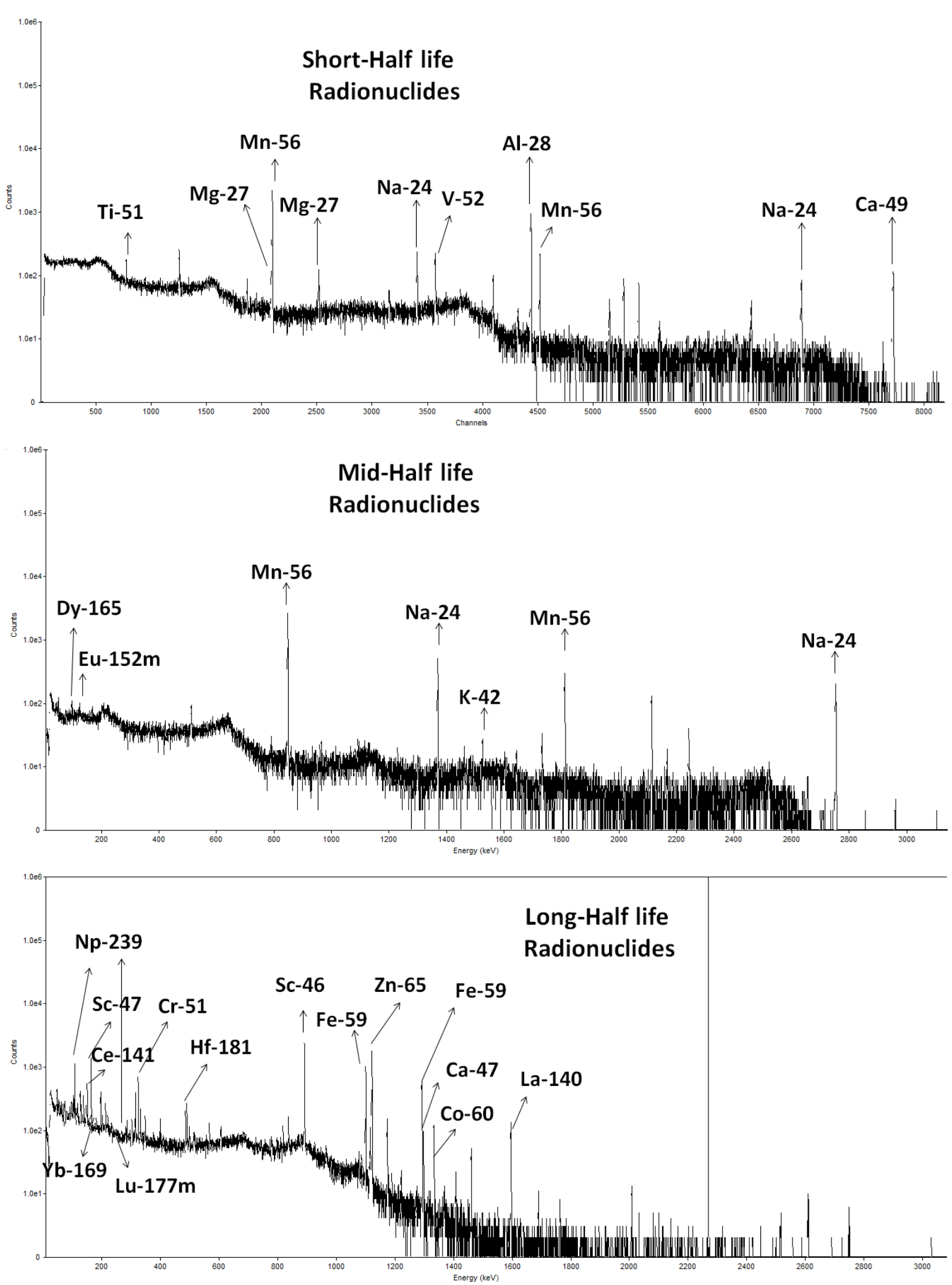

Fig.2 Gamma spectrums for short, mid and long half-life radionuclides in Abadan-Dec dust sample 


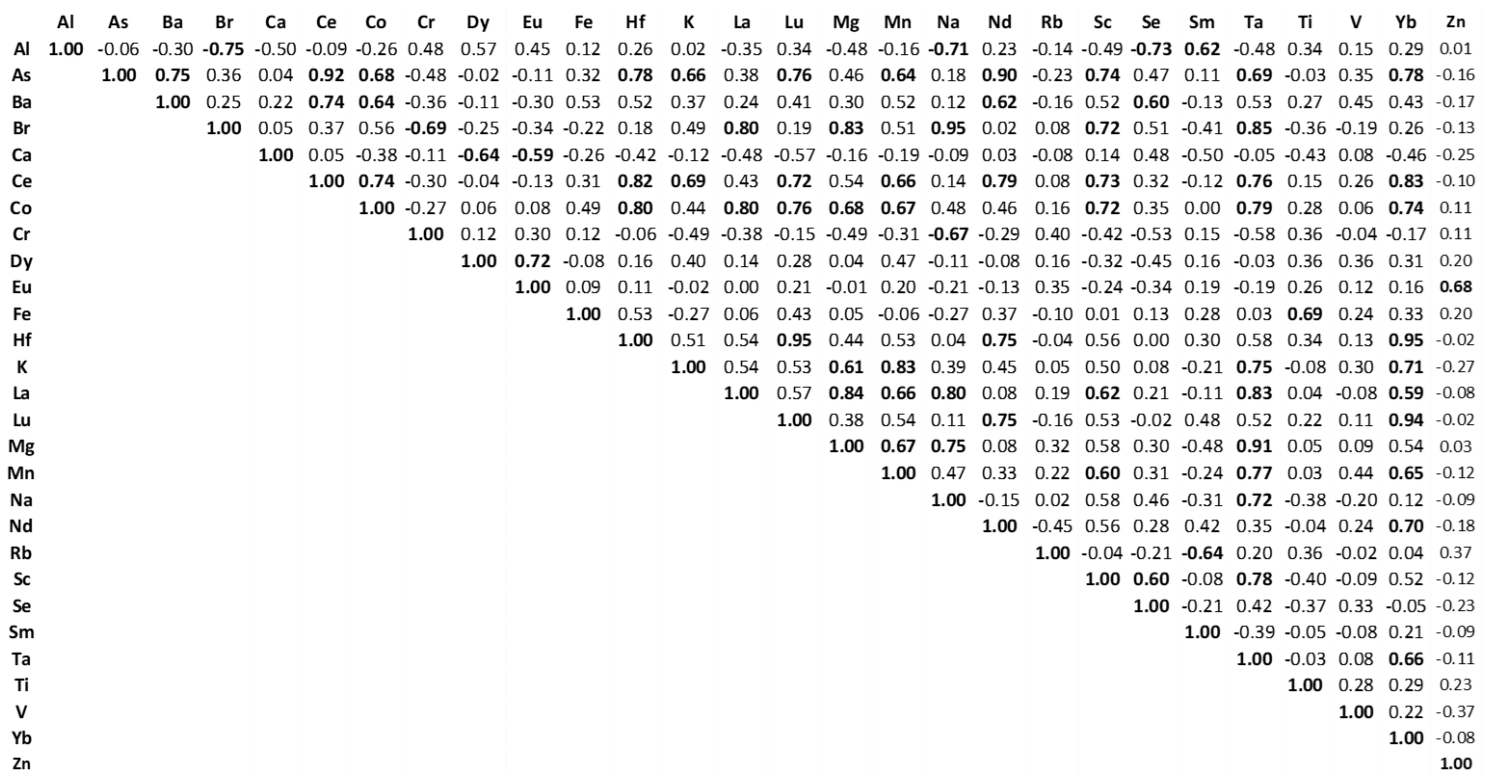

Fig.3 Correlations obtained for element concentrations in dust samle

\section{RESULTS AND DISCUSSION}

\section{A. INAA analysis}

The concentration of chemical elements in the dust sample resulting from INAA analysis are represented in (Table 2). The uncertainties are composed of a group of uncertainties such as sample weight, counting statistics, uncertainties reported for the concentration of radionuclides in the standard sample certificates. Because analysis with the SPAN software is based on a comparative method, uncertainties of efficiencies don't enter the composition of the resulting concentration uncertainty. There were many radionuclides with various half-lives in the samples. In practice, due to the numerous measurements in the gamma spectroscopy laboratory, planning to measure all of the radionuclides was not possible.

\section{B. Correlation among elements in the dust samples}

The correlations between the elements measured in the samples are given in (Fig 3). This quantity is divided into four categories. Very strong correlations with a coefficient greater than 0.8 , strong correlation with a coefficient in the range between 0.6 to 0.8 , medium correlation with a coefficient in the range between 0.4 to 0.6. Correlations with coefficients less than 0.4 are considered weak [37].In very strong correlations, different sources can be well distinguished from each other. Strong correlations between the elements in a category indicate the dependence of those elements on a single source [38, 39].

In (Fig 3), As and Se strongly correlate with the elements $\mathrm{Ce}$ and $\mathrm{Nd}$; $\mathrm{Nd}$ and $\mathrm{Ce}$ are among the rare earth elements whose most important production sources are oil and refining industries. Rare piles of earth along with As usually belong to fossil fuel combustion pollutants especially combined fuel power plants. $\mathrm{Br}$ has a strong correlation with the elements $\mathrm{La}, \mathrm{Mg}, \mathrm{Na}, \mathrm{Ta}$; as can be seen from the composition of the elements, the presence of terrestrial, sediment, and marine sources is well evident. $\mathrm{Na}$ and $\mathrm{Br}$, in correlation with each other, 
belong mainly to salt compounds derived from the sea or compounds from the bed of lakes or wetlands.

Co is strongly correlated with the elements $\mathrm{Hf}$, $\mathrm{Lu}, \mathrm{Mg}, \mathrm{Mn}, \mathrm{Sc}, \mathrm{Ta}, \mathrm{Yb}, \mathrm{Fe}, \mathrm{K}, \mathrm{Na}$; the compounds of cobalt, iron, manganese, potassium, sodium, and magnesium are related to the sources of the metal and steel industries. The rare piles of earth and Ta are also mainly dependent on the earth's crust. Ce is strongly correlated with the elements such as Hf, $\mathrm{Yb}, \mathrm{Co}, \mathrm{Lu}$; the main part of this composition is made of rare piles of earth. These elements are mainly used as catalysts in various industries such as oil and petrochemical industries and refineries. $\mathrm{Eu}$ and $\mathrm{Zn}$ also show strong correlations; $\mathrm{Eu}$ is mainly dependent on the earth's crust, $\mathrm{Zn}$ is dependent on waste, and in general, the correlation between the two can indicate the source of accumulation of waste in the bed of wetlands and rivers. Dy, Eu, Mn, Se are also mainly dependent on industrial wastes [40-49].

\section{Modeling with PMF technique and selecting the reference element for each sample}

To determine which element in each dust sample is the best choice as a reference element for $\mathrm{EF}$ calculations, the PMF (positive matrix factorization) modeling technique was used. This modeling is a computational approach to identify sources and determine their share in the samples based on their fingerprints. In this study, EPA-PMF 5.0 software has been used of the PMF to model [50].

\section{Table 4}

Selected reference element for each dust sample

\begin{tabular}{|c|c|c|c|c|}
\hline Sample name & $\begin{array}{c}\text { Reference } \\
\text { Material }\end{array}$ & $\begin{array}{l}\text { Identified anthropogenic source for } \\
\text { each dust sample by PMF }\end{array}$ & $\begin{array}{l}\text { Main Affected candidate } \\
\text { Reference elements in } \\
\text { Sample by the Identified } \\
\text { anthropogenic Sources }\end{array}$ & $\begin{array}{c}\text { Final } \\
\text { Selected } \\
\text { Reference } \\
\text { Element }\end{array}$ \\
\hline Abadan (23-Aug-18) & Soil & $\begin{array}{l}\text { See water-Sediment / Steel and } \\
\text { Metalworking }\end{array}$ & $\mathrm{Al}, \mathrm{Fe}, \mathrm{Sc}, \mathrm{Mn}$ & $\mathrm{Ti}$ \\
\hline Abadan (23-Sep-18) & Soil & $\begin{array}{l}\text { See water-Sediment / Waste/ Solid } \\
\text { Fuel }\end{array}$ & $\mathrm{Al}, \mathrm{Ti}, \mathrm{Sc}, \mathrm{Mn}$ & $\mathrm{Fe}$ \\
\hline Abadan (18-Dec-19) & Soil & Refinery / Steel and Metalworking & $\mathrm{Al}, \mathrm{Fe}, \mathrm{Sc}, \mathrm{Ti}$ & Mn \\
\hline Ahvaz & Soil & Waste/ Solid Fuel & $\mathrm{Al}, \mathrm{Ti}, \mathrm{Fe}, \mathrm{Sc}, \mathrm{Mn}$ & $\mathrm{Sc}$ \\
\hline Dezful & Soil & $\begin{array}{l}\text { Solid fuel/ Waste/ Steel and Metal } \\
\text { working }\end{array}$ & $\mathrm{Al}, \mathrm{Ti}, \mathrm{Fe}, \mathrm{Sc}, \mathrm{Mn}$ & Sc \\
\hline Hoveyzeh & Soil & Steel and Metalworking/ Solid Fuel & $\mathrm{Al}, \mathrm{Ti}, \mathrm{Fe}, \mathrm{Sc}, \mathrm{Mn}$ & $\mathrm{Sc}$ \\
\hline Mahshahr & Soil & $\begin{array}{l}\text { waste / Steel and Metal working/ see } \\
\text { water-Sediment (low) }\end{array}$ & $\mathrm{Al}, \mathrm{Fe}, \mathrm{Sc}, \mathrm{Mn}, \mathrm{Ti}$ & Sc \\
\hline Omidieh & Soil & $\begin{array}{l}\text { Solid Fuel/ Waste/ See water- } \\
\text { Sediment (very low contribution) }\end{array}$ & $\mathrm{Al}, \mathrm{Fe}, \mathrm{Ti}, \mathrm{Sc}, \mathrm{Mn}$ & Sc \\
\hline Ramhormoz & Soil & $\begin{array}{l}\text { Solid Fuel/ waste/ Steel and Metal- } \\
\text { working }\end{array}$ & $\mathrm{Al}, \mathrm{Fe}, \mathrm{Ti}, \mathrm{Sc}, \mathrm{Mn}$ & $\mathrm{Sc}$ \\
\hline Shush & Soil & $\begin{array}{l}\text { Solid Fuel/ Steel and Metal- } \\
\text { working/ }\end{array}$ & $\mathrm{Al}, \mathrm{Fe}, \mathrm{Ti}, \mathrm{Sc}, \mathrm{Mn}$ & Sc \\
\hline Susangerd & Soil & Waste/ Steel and Metalworking & $\mathrm{Al}, \mathrm{Fe}, \mathrm{Ti}, \mathrm{Sc}, \mathrm{Mn}$ & Sc \\
\hline
\end{tabular}


The obtained factors indicate the five sources, which include 1) steel and metalworking industries, 2) wastes, 3) saline compounds, and surface soil, which can mainly be the source of dried wetlands or sea sources, 4) oil-fired power plants and Mazut 5) oil refineries. The presence of these sources was also evident in the correlations found in (Fig. 3). Thus, according to the presence or absence of the mentioned sources in the dust samples, which shows the degree of influence from the pollution sources, among the five candidate reference elements $\mathrm{Sc}, \mathrm{Mn}, \mathrm{Ti}, \mathrm{Fe}$, and $\mathrm{Al}$, the most suitable one has been introduced for each dust sample in the (Table 4) $[34,36]$.

(Fig 4) shows the distribution of elemental concentrations corresponding to (Table 2). This figure consists of three parts. Section (a) indicates the distribution of $\mathrm{Br}, \mathrm{Na}, \mathrm{Nd}$, and $\mathrm{Zn}$ elements. Each of these distributions represents a source of pollutants, which was mentioned in the discussion of the correlations found among the elements (Fig 3). The highest concentrations of sodium and bromine were observed in Abadan, which could be evidence of the impact of the Persian Gulf, the Arvand River estuary, and the dried bed of the Hoor- Al-Azim wetland in the Azadegan plain due to the atmospheric currents. Neodymium, which represents the refinery source, had the highest concentration in Abadan, due to the Abadan refinery.

\section{Investigation of element concentrations in} samples and related biological hazards

In the case of Zinc, the area with the highest concentration was the Hoor-Al-Azim dried-up wetland; this may indicate the accumulation of industrial waste in that area.

Section (b) shows the distribution of metal element concentrations. Among the elemental concentrations obtained in (Table 2), the three metal elements, aluminum, iron, and magnesium, have large amounts of concentration. According to (Table 1), the LC50 quantities for these elements are over the limited values reported. In the case of iron, the cities of Ahvaz, Dezful, and Abadan show the highest concentrations, attributed to the large steel and metalworking industries in these cities. In Ahvaz, this quantity is more than 40 times the LC50. For magnesium, there is a concentration more than 13 times LC50 in Abadan due to the Abadan refinery.

The mineral element magnesium is one of the elements associated with rare piles of earth; in addition to existing in soil crust, rare piles of earth are among the pollutants produced by refineries. In the western and border towns such as Susangerd and Shush, Aluminum shows concentrations more than 20 times of LC50. The main source of these large amounts can be crustal soil, like the desert areas of the Azadegan plain. The aluminum industry and even war pollution in the neighboring 
country, Iraq, could be other possible sources. The rest of the elements in (Table 2) are mainly below the LC50 limits regarding respiratory hazards, which are not addressed here [13-18]. The results of the enrichment factor calculations are shown in (Fig 5).The soil reference material taken from the reference [49] has been used to perform the calculations. Reference elements used for each sample are indicated in (Table 4).

\section{E. The results of Enrichment factor calculations}

The classifications of obtained enrichment factors are such that the values smaller than 1 , like Ta, are dependent on the natural source of the earth's crust (soil). For $1<\mathrm{EF}<5$, causedhuman resources have been enrichment, which can be from small to medium values. For $5<\mathrm{EF}<20$, enrichment is very high.

Finally, for $20>E F$, the enrichment is very intense, such as the three elements Se in Ahvaz sample, $\mathrm{Br}$ in Abadan sample, and $\mathrm{Zn}$ in Susangerd sample. Comparison of (Fig 5) with results of correlations among elements in dust samples confirms the assessment made before.

For example, $\mathrm{EF}$ values of $\mathrm{Na}$ and especially $\mathrm{Br}$ in Abadan samples taken in Aguste, $\mathrm{Zn}$ values in Susangerd, $\mathrm{Fe}, \mathrm{Mn}, \mathrm{Cr}, \mathrm{Co}, \mathrm{Ti}, \mathrm{As}$, and especially Se in Ahvaz samples shows the effects of three pollutant sources mentioned before, including sea sources and sediments, waste deposited in dried bed of wetlands such as Hoor-Al-Azim and industrial sources of Ahvaz Such as metalworking. In addition, traces of other pollutant sources, such as solid fuel power plants and oil refineries, by investigation of $\mathrm{EF}$ values and making a comparison with elemental concentrations in (Table 2) and correlation values in (Fig 3) can be revealed. Altogether, these results show the elemental enrichment's dependence on pollutant sources scattered throughout the province, as stated in the description of correlations. 

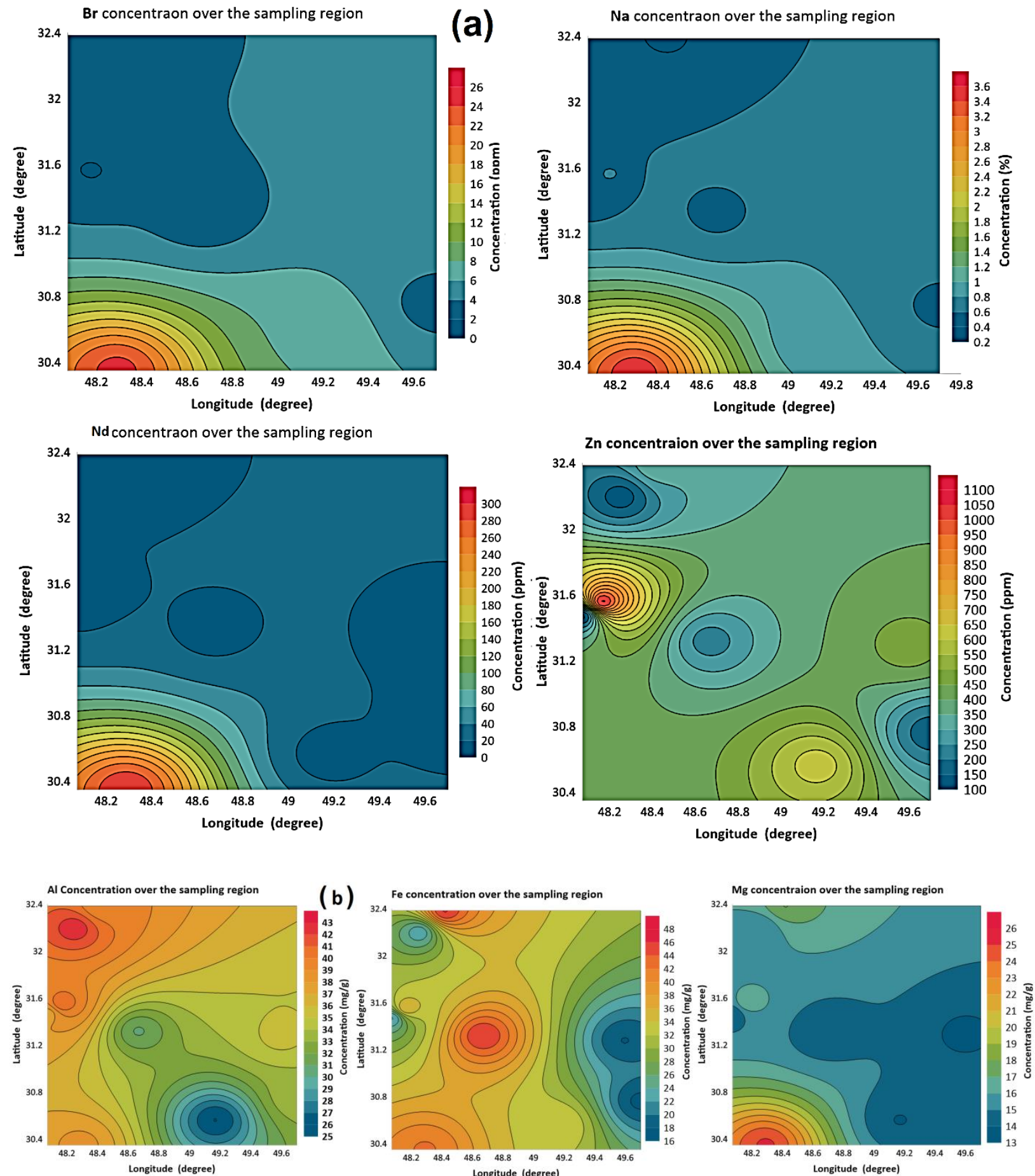

Fig.4. Distribution of Some measured elemental concentrations a) representative for some natural and anthropogenic sources (b) metallic elements with higher concentration values than LC50. The vertical axis indicates latitude (in degree) and the horizontal axis indicates longitude (in degree) of Khuzestan province plain. 


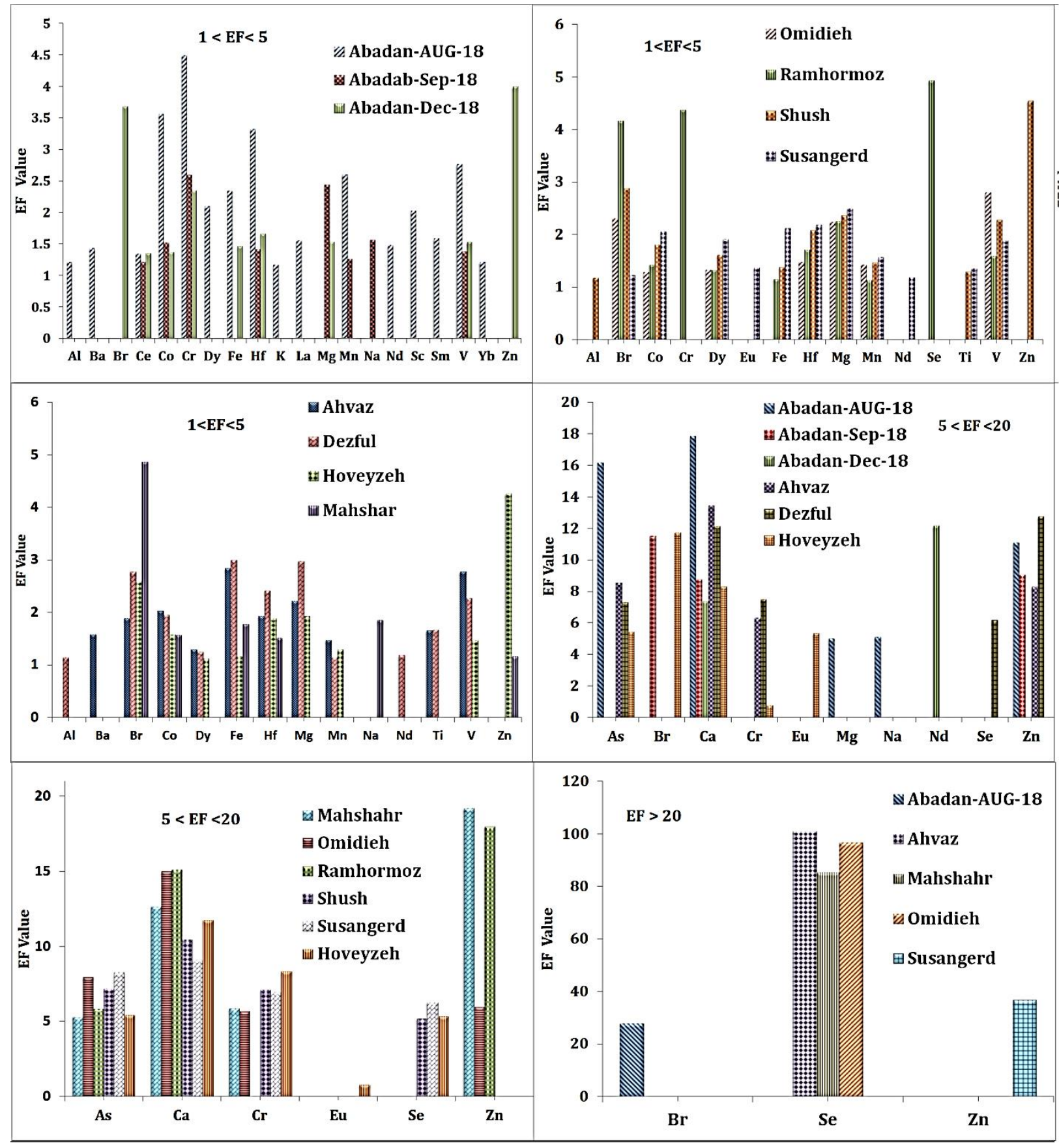

Fig. 5. Classification of elements according to the amount of EF calculated for dust samples of different cities

\section{Conclusion}

This study aimed to assess pollutant elements in air dust and investigate how they distribute following the occurrence of dust events in Khuzestan province and the changes in elemental concentrations of air ambient in different parts of the province. A comprehensive dust sampling was made over the areas covered with clay soil and are candidates for dust arising. Applying a new method to calculate the elements enrichment factor in the dust sample of each region based on the results of the PMF modeling and then 
evaluation of the results indicated that there is a direct relationship between the enrichment of elements and anthropogenic and natural pollutant sources distributed over the province. Dust storms are effective in the movements of pollutant particles all over the province. LC50 value for some metals such as iron, magnesium, and aluminum was much higher than the allowable values for these elements. This quantity for $\mathrm{Mg}$ in Abadan was above 25. The quentity of iron in Ahvaz, Shush, and Susangerd was higher than 40, and the value for aluminum in Susangerd and Shush was higher than 40. For zinc LC50, it was higher than 1000 , which is a great value.

The results showed very large enrichments with $\mathrm{EF}>20$ for elements such as $\mathrm{Zn}, \mathrm{Se}, \mathrm{Br}$ in Susangerd, Ahvaz, and Abadan. This value for Se was about 100, indicating high enrichment for this element and the impact of the industries in the province. The enrichment factors and the correlations between the elements in the samples of various sampling regions showed their dependence on local pollutant sources. Therefore, these results indicate the necessity for continuous monitoring of air particles, especially during and following dust storm.

\section{Acknowledgments}

We are so thankful to our colleagues in the Geological Survey of Iran for cooperation in the sampling process in this study.

\section{REFERENCES}

1. A. S. Goudie and N. J. Middleton, Desert Dust in the Global System, Springer, Berlin, Heidelberg, 1 th ed., 2006.

2. D. Francis, The dust load and radiative impact associated with the June 2020 historical Saharan dust storm, Atmos. Environ, 268, 118808 (2020)

3. P. Kulkarni,S. Chellam and M.P. Fraser, Lanthanum, and Lanthanides in Atmospheric Fine Particles and Their Apportionment to Refinery and Petrochemical Operations in Houston, TX, Atmos. Environ, 40(3), 508 (2006).

4. L. Gandois et al, Use of Geochemical Signatures, Including Rare Earth Elements, in Mosses and Lichens to Assess Spatial Integration and the Influence of Forest Environment, Atmos. Environ. 95, 96 (2014).

5. J.M. Lim et al,. Source apportionment of PM10 at a small industrial area using Positive Matrix Factorization, Atmos. Environ. 95 (1), 88 (2010).

6. P.G. Stegmann and P. Yang, A Regional, SizeDependent, and Causal Effective Medium Model for Asian and Saharan Mineral Dust Refractive Index Spectra, J. Aero. Sci., 14, 327 (2017).

7. B. Jancsek-Turóczi et al, Sampling and Characterization of Resuspended and Respirable Road Dust, Journal of Aerosol Science, 65, 69 (2013).

8. S.K. Song, Influence of Meteorological Conditions on Trans-Pacific Transport of Asian Dust During Spring Season, J. Aero. Sci. 39 (11), 1003 (2008)

9. B.E. Kim and M.K. Kim, Source Identification of Dust-Fall at Seashore in Korea by Positive Matrix Factorization, J. Aero. Sci. 35, S1147(2004).

10. J. Darvishi Khatooni, Composition and origin of foreign dust in Khuzestan province, 15, 93(2016)

11. ECHA, (European Chemical Agency), https://echa.europa.eu/registration-dossier//registered-dossier/15494/7/3/1, accessed in June 2021.

12. ECHA, (European Chemical Agency), https://echa.europa.eu/registration-dossier//registered-dossier/15551/7/3/1 , accessed in June 2021.

13. ECHA, (European Chemical Agency), https://echa.europa.eu/registration-dossier//registered-dossier/10184/7/3/1, accessed in June 2021.

14. ECHA, (European Chemical Agency), https://echa.europa.eu/registration-dossier//registered-dossier/15506/7/3/1, accessed in June 2021.

15. ECHA, (European Chemical Agency), https://echa.europa.eu/registration-dossier//registered-dossier/6440/7/3/1, accessed in June 2021.

16. ECHA, (European Chemical Agency), https://echa.europa.eu/registration-dossier//registered-dossier/16139/7/3/1, accessed in June 2021.

17. M. Talerko et al, Simulation Study of Radionuclide Atmospheric Transport after Wildland Fires in the Chernobyl, APR, 12(3), 193 (2020)

18. Z. Xu et al, Heavy metal pollution characteristics and health risk assessment of dust fall related to industrial activities in desert steppes, PeerJ, 9, $12430(2021)$

19. S. K. Pal, R. Roy, Evaluatiing heavy metals emission pattern on road influenced by urban layout, Transp, 10, 100362 (2021) 
20. M. Shirini et al, Pollution and contamination asenssment of heavy metals in the sediments of Jazmurian playa in southeat Iran, Sci Rep, 10, $4775(2020)$

21. S. Kumari, M. Kumar Jain, S. Pandian Elumalai, Assessment of Pollution and Health Risks of Heavy Metals in Particulate Matter and Road Dust Along the Road Network of Dhanbad, India, J. Health Pollut. 11(29): 210305

22. M. Barbieri, The Importance of Enrichment Factor (EF) and Geoaccumulation Index (Igeo) to Evaluate the Soil Contamination, J Geol Geophys. 5 (1) (2016).

23. G.C. Fang et al, Size Distributions of Ambient Air Particles and Enrichment Factor Analyses of Metallic Elements Taichung Harbor near the Taiwan Strait, Atmos. Res. 81(4), 320 (2006).

24. P. Kantor et al, Comparison of Enrichment Factors for Heavy Metals in Urban Street Dust and Air Aerosols, J. Pol. Miner. Eng. Soc. 19 (1), 206 (2018).

25. S. Dytlow and B. G. Kostrubiec, Concentration of Heavy Metals in Street dust: an Implication of Using Different Geochemical Background Data in Estimating the Level of Heavy Metal Pollution, Environ Geochem Health, 43, 521 (2021).

26. INTERNATIONAL ATOMIC ENERGY AGENCY, Sampling and Analytical Methodologies for Instrumental Neutron Activation Analysis of Airborne Particulate Matter, Training Course Series (1992)

27. C. Reimann, R.G. and Garrett, Geochemical Background-Concept and Reality, Sci. Total Environ. 350 (1-3), 12 (2005).

28. E. Triantafyllou et al, Contribution of LocallyProduced and Transported Air Pollution to Particulate Matter in a Small Insular Coastal City, APR, 11(4), 667 (2020).

29. A. Febo et al, Local air pollution and longrange mass transport of atmospheric particulate matter: A comparative study of the temporal evolution of the aerosol size fractions, APR , 1 (3), 141 (2010).

30. K. Lin, Seasonal Characteristics of PM2.5 and its Chemical Species in the Northern Rural China. APR, 11, 1891 (2020).

31. W. Javed and B. Guo, Chemical characterization and source apportionment of fine and coarse atmospheric particulate matter in Doha, Qatar, APR, 12 (2), 122 (2021).

32. R. Zhao et al, Element Composition and Source Apportionment of Atmospheric Aerosols over the China Sea, APR, 6 (2), 191(2015)

33. W.H. Zoller, E.S. Gladney, and R.A. Duce, Atmospheric Concentrations and Sources of trace Metals at the South Pole, Science. 183, 198 (1974).

34. S.R. Taylor, Abundance of Geochemical Elements in the Continental Crust: A New Table, Geochemical et Cosmochimca Acta, 28 (8), 1273 (1964)
35. L. Lucadamo and L.G.A. Corapi, Power plants: The Need for Effective Bio monitoring of the Contribution of Bio (Wood) Fuelled Stations to Atmospheric Contamination, APR, 10 (6), 2040 (2019).

36. R.B. Carleton, K. Walton-Day and D.L. Naftz, Improved enrichment factor calculations through principal component analysis: Examples from Soils Near Breccia Pipe Uranium Mines, Arizona, USA, Environ. Pollut. 248, 90 (2019)

37. H. Akoglu, User's Guide to Correlation Coefficients. Turk J Emerg Med, 18 (3), 91(2018).

38. S.J. Ramos, et al, Rare Earth Elements in the Soil Environment, Curr Pollution Rep, 2, (28) (2016).

39. M. Edahbi et al, Rare Earth Elements (La, Ce, $\mathrm{Pr}, \mathrm{Nd}$, and $\mathrm{Sm}$ ) from a Carbonatite Deposit: Mineralogical Characterization and Geochemical Behavior, Minerals, 8 (55) (2018).

40. Y. Arfala et al, Assessment of Heavy Metals Released into the Air from the Cement Kilns Co-Burning Waste: Case of Oujda Cement Manufacturing (Northeast Morocco), SER, 28, 363 (2108)

41. R. Meij, Trace Element Behavior in Coal-Fired Power Plants, FPT. 30 (1-3), 199 (1994)

42. F. A. Atikulet al, Comparative Test Analysis of Petroleum (Diesel and Gasoline) Soots as Potential Sources of Toxic Metals from Exhausts of Power Plants, Scholars Research Library, Arch Appl Sci Res, 3 (4), 147 (2011).

43. J.A. Caruso et al, Petroleum Coke in the Urban Environment: A Review of Potential Health Effects. IJERPH, 29, 12 (6), 6218, (2015).

44. T. Thi et al, Release of Trace Elements from Bottom Ash from Hazardous Waste Incinerators. Recycling. 3, 36 (2018).

45. A. Font, Using Metal Ratios to Detect Emissions from Municipal Waste Incinerators in Ambient Air Pollution Data, Atmos. Environ, 113, 177 (2015).

46. Lee B Clarke, The Fate of Trace Elements During Coal Combustion and Gasification: an Overview, Fuel, 72 (6), 731(1993).

47. Q.L. Dai et al, Characterization and Source Identification of Heavy Metals in Ambient PM10 and PM2.5 in an Integrated Iron and Steel Industry Zone Compared with a Background Site. AAQR, 15, 875(2015).

48. A. González and R. Navia Fly Ashes from Coal and Petroleum Coke Combustion: Current and Innovative Potential Applications, Waste Manag Res, 27, 976 (2009).

49. L. H. Ahrens, Origin and Distribution of the Elements, 1th Edition (International Series of Monographs in Earth Sciences, 1968).

50. EPA-PFF 5.0. United State Environmental Protection Agency https://www.epa.gov/airresearch/positive-matrix-factorization-modelenvironmental-data-analyses

How to cite this article

Z. Akbari, O. Kakuee, R. Shahbazi, et al, Assessment of pollutant elements content in ambient air dust of Khuzestan province, Journal of Nuclear Science and Applications 2 (1): 45-60 (2022), DOI: 10.24200/jon.2022.1012 